\title{
TEM Sample Preparation of Ceramic Matrix Composites Using FIB
}

Shannon Poges ${ }^{1}$, Jacqueline E. Cloud ${ }^{1}$, Mark Aindow ${ }^{2,3}$ and Steven L. Suib ${ }^{1,2,3,4}$

1. Dept. of Chemistry, University of Connecticut, Storrs, CT, USA.

2. Dept. of Materials Science and Engineering, University of Connecticut, Storrs, CT, USA.

3. Institute of Materials Science, University of Connecticut, Storrs, CT, USA.

4. Dept. of Chemical \& Biomolecular Engineering, University of Connecticut, Storrs, CT, USA.

Ceramic Matrix Composites (CMCs) combine the toughness of advanced composites with the high temperature functionality, oxidation resistance and mechanical strength of ceramic materials. These multi-component composites are capable of operating in extreme conditions such as those in power generation, aerospace, and aircraft technologies [1,2]. CMCs are typically composed of three components: reinforcing fiber, interphase, and matrix. The two major classifications of these multicomponent composites are oxide and non-oxide. Oxide CMCs are made with various metal oxide components allowing the $\mathrm{CMC}$ to be used in high temperature oxidizing environments without compromising the strength of the material due to unwanted oxidation. Some examples of ceramics used in oxide CMCs are alumina, silica, boria, zirconia and yittria [3]. Non-oxide CMCs can be composed of but are not limited to silicon carbide, boron nitride, titanium diboride, silicon nitride, and carbon [2].

The materials considered in this study are miniature CMCs composed of a single Sylramic ${ }^{\mathrm{TM}} \mathrm{SiC}$ fiber tow, chemical vapor deposition (CVD) applied BN interphase layer, and a CVD/polymer SiC matrix. We have developed a sample preparation method using the latest generation of dual-beam $\mathrm{Ga}^{+}$FIB instruments (FEI Helios Nanolab G3) which can lead to extraction of the maximum TEM data from a single CMC TEM sample. In the example shown in Figure 1, a sample site was chosen that included two coated fibers $(20 \mu \mathrm{m})$, with good penteration of matrix material between the fibers. A differential thinning pattern was adopted, with one fiber/interphase area thinned to 50-60 $\mathrm{nm}$ to give a parallel-sided lamella suitable for elemental analysis, whereas the other was thinned to perforation to give regions around the holes suitable for HRTEM lattice imaging. Typical FIB lift out procedures were employed to create the lamella. Since the $\mathrm{SiC}$ fibers mill at a lower rate than the interphase layer or the matrix, the milling was repeated over the same area to ensure uniform cross sectioning to a depth of about $7 \mu \mathrm{m}$ (Figure 1a). Once the lamella was transferred to the $\mathrm{Cu}$ grid, the central region was thinned to electron transparency $(<60 \mathrm{~nm})$ using a flip stage to obtain STEM images at $30 \mathrm{kV}$ to monitor Pt removal and hole formation. Examples of data obtained from this sample in an FEI Talos F200X TEM/STEM are shown in Figures 2 and 3. Elemental maps from the thicker area reveal the compositional distribution very clearly, and there is also strong uniform contrast in HAADF STEM images from this region. HRTEM lattice images from the thinnest areas around the hole reveal that the phases present in the matrix and interphase are cubic $\beta$-SiC and hexagonal BN. These data illustrate how a range of different types of TEM experiments can be performed on a single FIB-cut sample with this geometry. [4]

References:

[1] Jaques Lamon in "Ceramic Matrix Composites: Materials, Modeling and Technology", ed. 1, Narottam P. Bansal, (Wiley, Hoboken) p. 3.

[2] F. C. Campbell, in "Structural Composite Materials", (ASM International, Materials Park) p. 2.

[3] K. A. Keller in "Oxide-Oxide Composites, Ceramic Matrix Composites: Materials, Modeling and Technology",ed. 1, Narottam P. Bansal, (Wiley, Hoboken) p. 236. 
[4] This work was supported in part by a research grant from FEI Company under an FEI-UConn partnership agreement. The studies were performed in the UConn/FEI Center for Advanced Microscopy and Materials Analysis (CAMMA).

a.
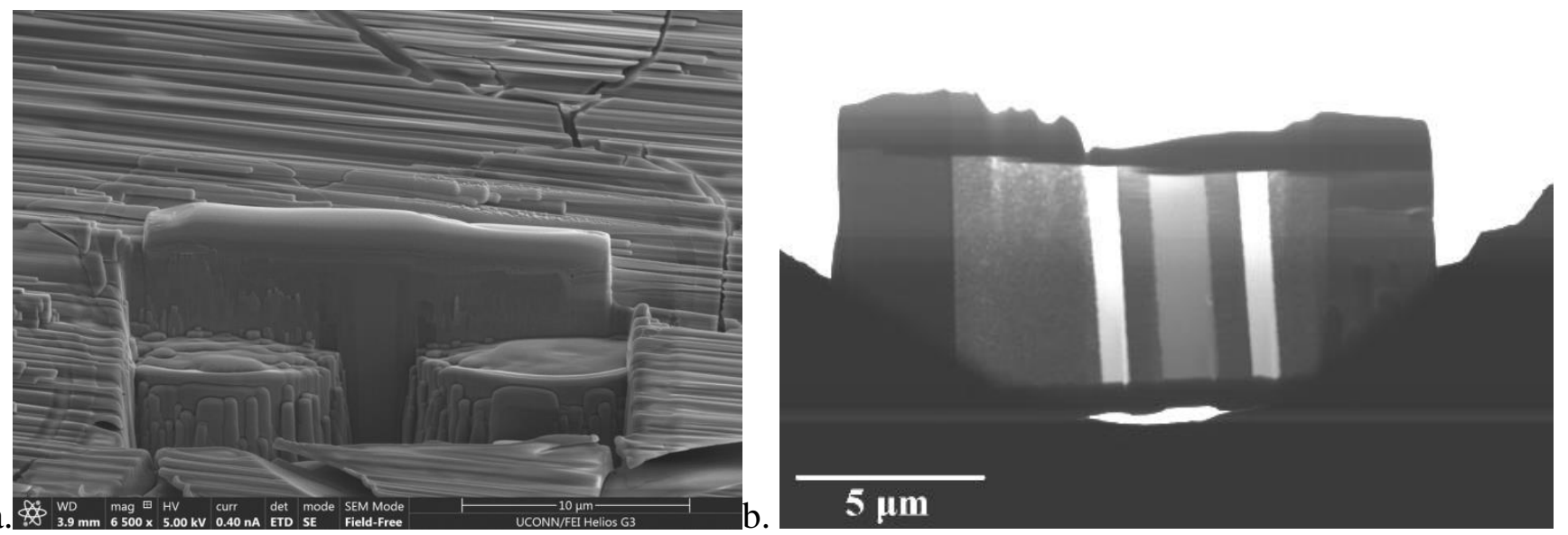

Figure 1. SEM micrographs of: (a) CMC sample during milling; (b) FIB-cut lamella with Pt cap. (left to right: $\mathrm{SiC}$ fiber, CVD BN interphase, CVD SiC , SiC Matrix, CVD SiC, CVD BN interphase, SiC fiber.)

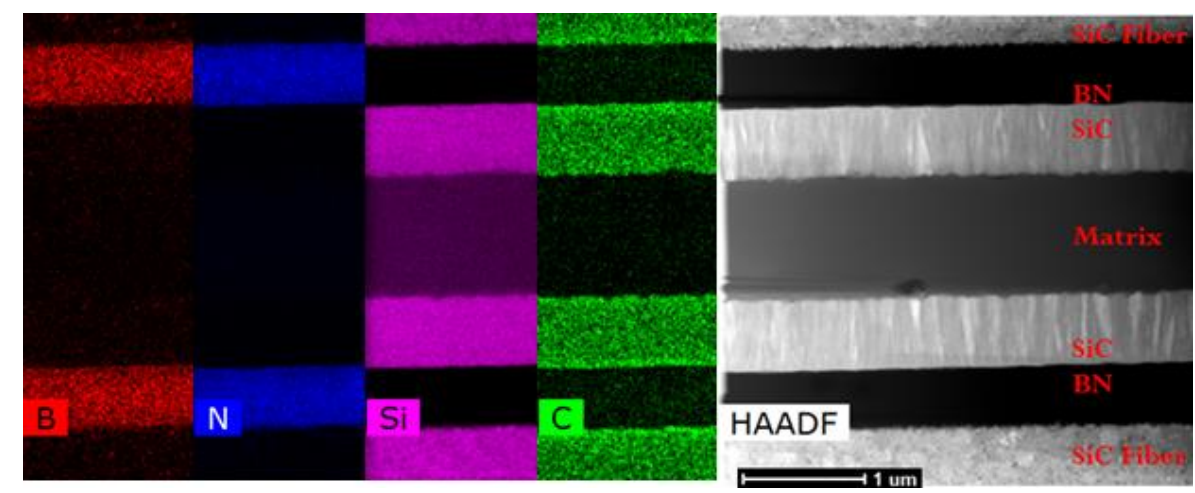

Figure 2. EDXS elemental maps and HAADF-STEM image from the FIB-cut CMC lamella in Fig 1(b).

a.

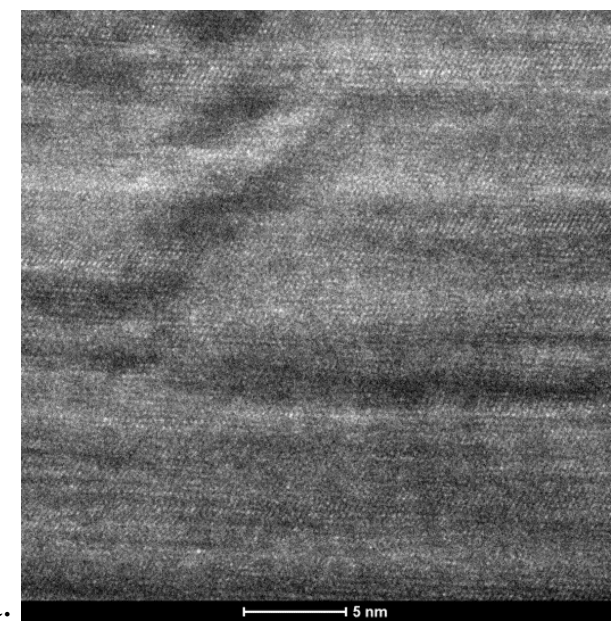

b.

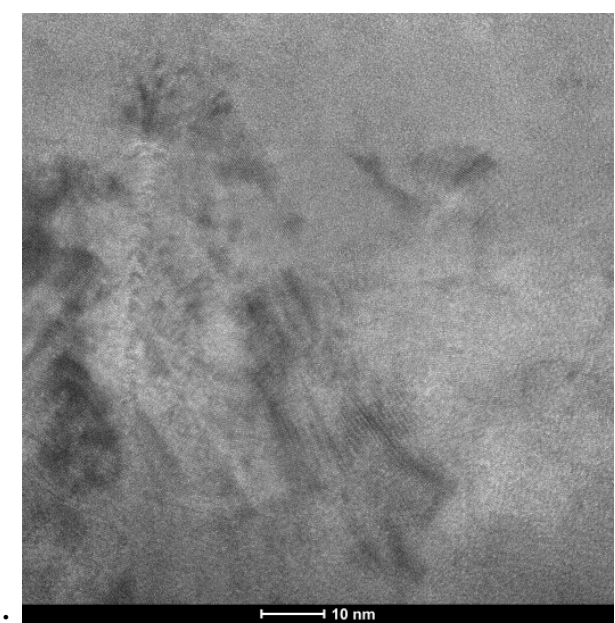

Figure 3. HRTEM micrographs revealing regions of (a) $\beta$-SiC and (b) h-BN CVD coatings. 